\title{
Ionic Liquid-Assisted Synthesis of a NiO/CNTs Composite and Its Electrochemical Capacitance
}

\author{
Yitong Chen, ${ }^{1}$ Xiangjun $\mathrm{Lu}^{2}$ and Bo Gao ${ }^{3}$ \\ ${ }^{1}$ Xinjiang Institute of Ecology \& Geography, Chinese Academy of Sciences, Urumqi 830011, China \\ ${ }^{2}$ Electronic Components Testing Center, The Fifth Electronics Research Institute of Ministry of Industry \& Information Technology, \\ Guangzhou 510610, China \\ ${ }^{3}$ Key Laboratory of Functional Materials \& Devices under Special Environments, \\ Xinjiang Key Laboratory of Electronic Information Materials \& Devices, Xinjiang Technical Institute of Physics \& Chemistry, \\ Chinese Academy of Sciences, Urumqi, Xinjiang 830011, China
}

Correspondence should be addressed to Bo Gao; gaobo@ms.xjb.ac.cn

Received 30 September 2014; Revised 1 December 2014; Accepted 1 December 2014; Published 25 December 2014

Academic Editor: Alan K. T. Lau

Copyright (C) 2014 Yitong Chen et al. This is an open access article distributed under the Creative Commons Attribution License, which permits unrestricted use, distribution, and reproduction in any medium, provided the original work is properly cited.

\begin{abstract}
A novel solid-state method has been developed for synthesizing nickel oxide (NiO)/carbon nanotubes (CNTs) composite using an ionic liquid (IL, 1-butyl-3-methylimidazolium chloride) as the reaction medium. Ultraviolet-visible (UV-vis) absorbance spectroscopy, infrared spectroscopy (IR), and scanning electron microscopy (SEM) were employed to investigate the structure, morphology, and formation mechanism of the synthesized sample. The results demonstrated that the IL is effective for dispersing CNTs, which allows the tethering of nickel (II) ions onto the surfaces of the CNTs and facilitates the subsequent chemical deposition of $\mathrm{NiO}$ to obtain the $\mathrm{NiO} / \mathrm{CNTs}$ composite. The electrochemical properties of the composite were determined using cyclic voltammetry and galvanostatic charge/discharge measurements in $6 \mathrm{M} \mathrm{KOH}$. Because of its unique structure, the prepared $\mathrm{NiO} / \mathrm{CNTs}$ electrode exhibited good capacitive behavior and cyclability. The high specific capacitance $\left(521 \mathrm{~F} \mathrm{~g}^{-1}\right)$ and good rate capability $\left(91 \%\right.$ capacity retention at $0.5 \mathrm{~A} \mathrm{~g}^{-1}$ ) of the $\mathrm{NiO} / \mathrm{CNT}$ composite enable its use as a practical supercapacitor electrode material.
\end{abstract}

\section{Introduction}

Growing environmental concerns and the increasing depletion of fossil fuels have created interest in alternative energy technologies. Electrochemical capacitors (ECs), which are energy storage devices with properties between those of batteries and electrostatic capacitors, possess several desirable properties, such as high power density (ten times greater than that of batteries), rapid charging within seconds, and excellent cycling stability [1-3]. These properties make ECs one of the most promising candidates for next-generation power devices [4]. With characteristics that complement those of rechargeable batteries and fuel cells, ECs have been used in many applications, such as backup power supplies, pacemakers, air bags, and electric vehicles [4-6]. ECs can generally be classified into two types depending on the charge storage mechanism and on the active materials used. The first type includes electrochemical double-layer capacitors based on carbon materials, in which the charge is stored between the electrode and the electrolyte [5-7]. The second type includes pseudocapacitors with transition metal oxides or conductive polymers, in which the capacitance is produced through rapid faradaic reactions, such as intercalation, underpotential deposition, or redox processes that occur at or near the surface of a solid electrode at an appropriate potential [5, 7]. Among the active materials used in ECs, nickel oxide $(\mathrm{NiO})$ has received considerable attention for use in pseudocapacitors due to its low cost, environmentally benign nature, easy synthesis, and high theoretical capacity value (ca. $2573 \mathrm{~F} \mathrm{~g}^{-1}$ ) [8]. However, due to its inability to support rapid electron and electrolyte ion transport, $\mathrm{NiO}$ suffers from a low rate capability and capacity, which is only one-third or one-half of the theoretical value [8]. In other words, the capacitance is greatly limited by the surface reaction and is 




SCHEme 1: Schematic diagram of the procedure for synthesizing the NiO/CNTs composite.

unable to meet the increasing demands for the development of energy storage technologies and the application ranges of various electrolytes. Many previous studies have shown that combining $\mathrm{NiO}$ with carbon materials that possess high specific surface areas can improve the electrochemical performance of $\mathrm{NiO}[9,10]$.

Because of their chemical stability, good conductivity, and large surface area, carbon nanotubes (CNTs) are considered a good carbon material for combination with $\mathrm{NiO}$. Recently, CNT-based NiO composites have been investigated. These composites are expected to exhibit improved capacitance due to the interaction between $\mathrm{NiO}$ and CNTs $[9,10]$. However, loading $\mathrm{NiO}$ onto the surface of the CNTs is difficult due to the intact and smooth surface of the CNTs and the poor solubility of the CNTs in solvents due to van der Waals interactions. Therefore, considerable efforts have been made towards functionalizing CNTs [9-12]. Traditionally, CNTs were modified or functionalized with different types of functional groups through reactions on the $\pi$-conjugated skeleton of the CNTs. Unfortunately, this covalent binding site primarily occurs at defect sites or at the ends of CNTs. Consequently, the $\mathrm{C}$ atoms in the CNTs transform from $\mathrm{sp}^{2}$-hybridized to $\mathrm{sp}^{3}$-hybridized $\mathrm{C}$ atoms. However, this transform leads to the problem of electron transfer not only along the two-dimensional (2D) surface of CNTs but also transfer between the additional components during the charge/discharge process [11]. The destruction of the carbon bands of covalently functionalized CNTs will result in undesirable compromises in the electronic and mechanical properties, such as a decrease in the conductivity. Moreover, the utilization of covalently modified CNTs may result in inhomogeneous coverage of additional components that tend to attach at the ends and defect sites of the CNTs, where the concentrations of the functional moieties are the highest [9-12]. Therefore, noncovalent functionalization of CNTs is becoming particularly attractive because it can be used to attach chemical handles without affecting the electronic network of the tubes. This noncovalent interaction is based on van der Waals forces or $\pi-\pi$ stacking and is thermodynamically controlled. The dispersion and homogeneity of electroactive materials improve when they are loaded in high amounts on CNTs [10].

Therefore, we developed a general strategy for synthesizing two-dimensional (2D) NiO/CNTs nanotubular electrode materials using an imidazolium ionic liquid (IL) as the reaction medium. The IL plays a dual role during (2D) $\mathrm{NiO} / \mathrm{CNTs}$ synthesis. First, the dispersion of CNTs is improved through $\pi-\pi$ conjugate actions between the $\pi$ electrons on the surface of the CNTs and the IL [13]. Second, the intramolecular hydrogen bond between the anion and cation of the IL ensures that the $\mathrm{NiO}$ is uniformly dispersed on the surface of the CNTs. Electrochemical tests show that the $\mathrm{NiO} / \mathrm{CNT}$ composites prepared using this method have enhanced specific capacitance and rate performance.

\section{Experimental Details}

2.1. Synthesis of the $\mathrm{NiO} / \mathrm{CNTS}$ Composite. $\mathrm{NiCl}_{2} \cdot 6 \mathrm{H}_{2} \mathrm{O}$ and solid potassium hydroxide $(\mathrm{KOH})$ were used as analytical reagents. In addition, the 1-methylimidazole (98.0\%), 1-butyl chloride, and dehydrated solvents $\left(\mathrm{H}_{2} \mathrm{O}<50 \mathrm{ppm}\right)$ were purchased from WAKO Pure Chemical Industries, Ltd. The CNTs were purchased from Nanotech Port Co., Ltd. (Shenzhen, China) and were purified by refluxing in concentrated hydrochloric acid $(\mathrm{HCl})$ at $313 \mathrm{~K}$ for $6 \mathrm{~h}$ prior to use (denoted as CNTs) $[14,15]$.

The synthetic route for obtaining the NiO/CNTs composite is shown in Scheme 1. In the first step of this method [15], a mixture of 3-methylimidazole and 1-butyl chloride was degassed using three freeze-pump-thaw cycles followed by purging with nitrogen. The salt $[\mathrm{Bmim}] \mathrm{Cl}(1)$ was obtained by reacting 3-methylimidazole and 1-butyl chloride. Salt 1 was purified by recrystallization from an acetonitrile solution at $263 \mathrm{~K}$ to remove the yellow color of the initial crude product. In the next step, 1 was reacted with the metal chloride $\mathrm{NiCl}_{2} \cdot 6 \mathrm{H}_{2} \mathrm{O}$ without solvent at 298 and $373 \mathrm{~K}$ for $24 \mathrm{~h}$ and 
then reacted in acetonitrile at $363 \mathrm{~K}$, which afforded the metal oxide precursor Bmim $\left[\mathrm{NiCl}_{3}\right]$. After the desired precursors were obtained, the CNTs were introduced using a solid-state strategy. First, the CNTs and $\mathrm{Bmim}\left[\mathrm{NiCl}_{3}\right]$ were mixed at a certain mass ratio by ball milling. Next, the mixture was transferred to a ball mill containing a certain amount of $\mathrm{KOH}$. After ball milling for $2 \mathrm{~h}$, the product was naturally cooled to room temperature. The black precipitate, which had a $\mathrm{NiO}$ mass loading of $10 \mathrm{wt} . \%$ based on the calculated weight of the CNTs, was collected after centrifugal filtration by washing with deionized water and ethanol and drying under vacuum for $24 \mathrm{~h}$ at $378 \mathrm{~K}$. Then, the samples were placed in a muffle furnace and calcined at $573 \mathrm{~K}$ for $2 \mathrm{~h}$ at a ramp rate of $1 \mathrm{Kmin}^{-1}$, which transformed the samples into the $\mathrm{NiO} / \mathrm{CNTs}$ composite. For comparison, $\mathrm{NiO}$ was prepared using $\mathrm{Bmim}\left[\mathrm{NiCl}_{3}\right]$ and $\mathrm{KOH}$ under the same reaction conditions.

2.2. Material Characterizations. Fourier transform infrared (FTIR) spectra of the CNTs, $\mathrm{Bmim}\left[\mathrm{NiCl}_{3}\right]$, and CNTs/ $\mathrm{Bmim}\left[\mathrm{NiCl}_{3}\right]$ suspensions were recorded between 3750 and $500 \mathrm{~cm}^{-1}$ using a Bruker FTIR spectrophotometer. In addition, UV-vis spectra were recorded between 400 and $650 \mathrm{~cm}^{-1}$ using a Cary 5G UV/vis-NIR spectrometer (Varian). Prior to measurement, each sample was diluted to onefifth of its initial concentration using distilled water. X-ray diffraction (XRD) measurements were performed using a Bruker D8 Advance-X diffractometer with a $\mathrm{Cu} \mathrm{K} \alpha$ source. Scanning electron microscopy (SEM) was performed using a Zeiss Supra 55.

\subsection{Electrode Preparation and Electrochemical Measure-} ments. The sample electrodes were fabricated by mixing and grinding $80 \mathrm{wt} . \%$ sample with $15 \mathrm{wt} . \%$ carbon black and 5 wt.\% polytetrafluoroethylene (PTFE) before pressing onto a graphite substrate. The graphite support was first abraded with ultrafine $\mathrm{SiC}$ paper, rinsed in an ultrasonic water bath for 15 min, etched in a $1 \mathrm{M} \mathrm{H}_{2} \mathrm{SO}_{4}$ solution at room temperature for $10 \mathrm{~min}$, and rinsed in an ultrasonic water bath for $30 \mathrm{~min}$. The exposed geometric area of the treated graphite support was $1 \mathrm{~cm} \times 1 \mathrm{~cm}$. The mass of the active material on each electrode was $5 \mathrm{mg}$. All electrochemical measurements were conducted using a three-electrode system, with a $\mathrm{NiO} / \mathrm{CNTs}$ or $\mathrm{NiO}$ electrode, platinum foil, and a standard calomel electrode (SCE) as the working, counter, and reference electrodes, respectively. A $6 \mathrm{M} \mathrm{KOH}$ aqueous electrolyte solution was used. The cyclic voltammetry (CV) and galvanostatic charge/discharge tests were performed using a CHI660B electrochemical workstation (Chenhua, China).

\section{Results and Discussion}

Scheme 1 illustrates the potential mechanisms for the synthesis of the $\mathrm{NiO} / \mathrm{CNTs}$ composite. The IL [Bmim] Cl was selected as the reaction medium because the imidazolium ions likely interact with the $\pi$-electron network of the CNTs through a "cation- $\pi$ " interaction [10]. The following steps were used: first, the IL salt $\mathrm{Bmim}\left[\mathrm{NiCl}_{3}\right]$ was dispersed and absorbed onto the surface of the CNTs through $\pi$ $\pi$ interactions during the milling process. Simultaneously, $\mathrm{Ni}^{2+}$ was concentrated on the surface of the CNTs using static electricity between the anion, cation, and hydrogen bonds of the IL molecules, thereby facilitating a uniform $\mathrm{Ni}^{2+}$ distribution. Second, $\mathrm{KOH}$ could react with $\mathrm{Bmim}\left[\mathrm{NiCl}_{3}\right]$ to generate $\mathrm{Ni}(\mathrm{OH})_{2}$ on the surface of the CNTs. After washing, drying and calcining, the $\mathrm{NiO} / \mathrm{CNTs}$ composite was obtained via in situ coating induced by the strong electron interactions between the electron-deficient benzene ring of the CNTs and the electron-rich imidazole ring of the IL. Generally, ILs act as not only "bridges" to connect $\mathrm{Ni}^{2+}$ ions onto the surfaces of CNTs but also as "hosts" to accommodate and stabilize the $\mathrm{NiO}$ generated in situ [15].

The obtained $\mathrm{CNTs} / \mathrm{Bmim}\left[\mathrm{NiCl}_{3}\right]$ liquid was stored at room temperature for 1 week to test its stability. Photographs of the dispersions are shown in Figure 1(a) (I II III IV). Based on our investigations the color and concentration of the $\mathrm{CNTs} / \mathrm{Bmim}\left[\mathrm{NiCl}_{3}\right]$ sols (IV) remain consistent regardless of the storage time [16-20]. The unmodified or noncovalently functionalized CNTs have fewer functional groups, which could prevent chain entanglement and agglomeration in the ILs. Thus, better dispersion of the $\mathrm{CNTs} / \mathrm{Bmim}\left[\mathrm{NiCl}_{3}\right]$ sols occurs when fewer functional groups are present on the CNTs $[16,17]$. However, due to the tight $\pi$-stacking interactions between the relatively electron-rich imidazole ring of the IL and the electron-deficient benzene ring of the CNTs, the IL is strongly adsorbed on the surface of the CNTs. Simultaneously, the surfaces of the CNTs were enriched with $\mathrm{Cl}^{-}$ anions due to the intramolecular hydrogen bonding of the IL. The CNTs repelled each other through electrostatic forces. Consequently, the CNTs were well dispersed in the IL $[16,17$, 21], as indicated by the FTIR spectra. As shown in Figure 1(b), the spectra from the CNTs were normalized with respect to the $\mathrm{C}-\mathrm{H}$ bands at $2800-3000 \mathrm{~cm}^{-1}$. This normalization was attributed to the symmetric and antisymmetric stretching vibrations of $\mathrm{CH}_{2}[16,17,21]$. Because the CNTs do not support a static dipole moment, a relatively weak dynamic dipole moment was responsible for the observed infraredactive modes of the CNTs. Thus, the characteristic IR absorption peaks of the CNTs were very weak. The observed spectra from the $\mathrm{CNTs} / \mathrm{Bmim}\left[\mathrm{NiCl}_{3}\right]$ solution only resulted from the $\mathrm{Bmim}\left[\mathrm{NiCl}_{3}\right]$. The bands in the $3011-3196 \mathrm{~cm}^{-1}$ region are assigned to the $\mathrm{C}-\mathrm{H}$ stretching vibration modes of the imidazolium ring $[16,17]$. The unresolved $\mathrm{CH}_{3}-\mathrm{N}$ stretching vibration, the $\mathrm{CH}_{2}-\mathrm{N}$ stretching vibration, and the imidazolium ring in-plane asymmetric stretching bands appeared at 926 and $1502 \mathrm{~cm}^{-1}$ [16]. Compared with the spectrum of the CNTs, the characteristic bands for the $\mathrm{CNTs} / \mathrm{Bmim}[\mathrm{Ni}]^{3+}$ solution appear in the range of $700-1600 \mathrm{~cm}^{-1}$ [15-17], showing nearly identical numbers and positions as those of the main peaks from the CNTs. The cation- $\pi$ or $\pi-\pi$ stacking between the CNTs and $\mathrm{Bmim}\left[\mathrm{NiCl}_{3}\right]$ altered the $\mathrm{C}=\mathrm{C}$ and $\mathrm{C}=\mathrm{N}$ bond lengths, which caused the peaks to shift slightly from $1564 \mathrm{~cm}^{-1}$ to $1569 \mathrm{~cm}^{-1}$ [19]. To further illustrate the cation- $\pi$ and $\pi-\pi$ conjugate force, the UV-vis spectra are shown in Figure 1(c). Here, the curve for the CNTs 


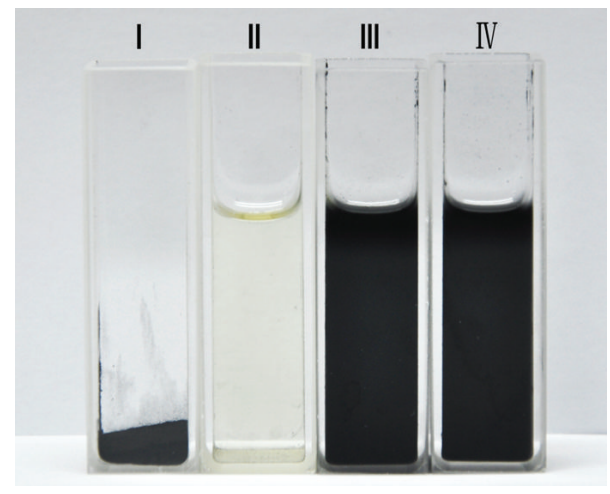

(a)

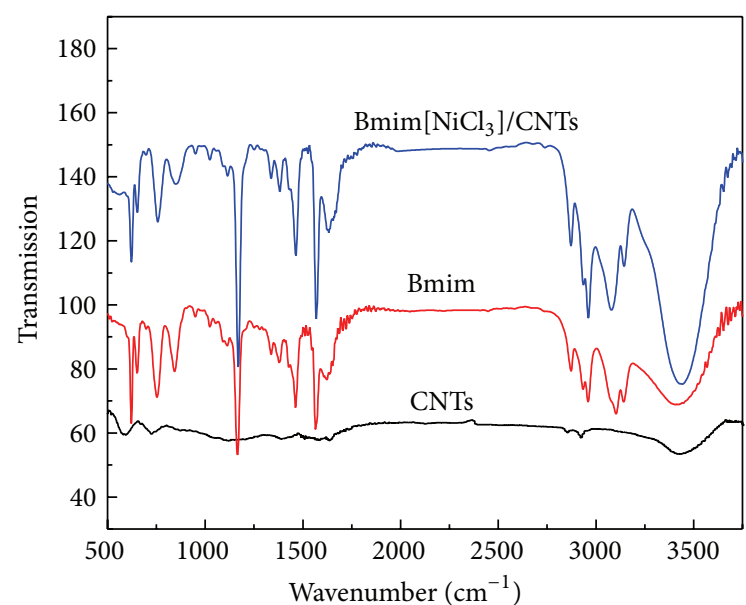

(b)

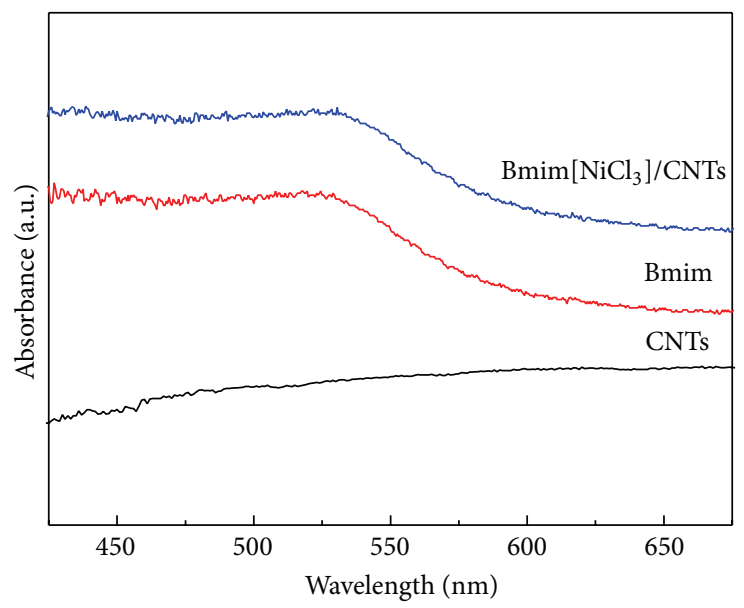

(c)

FIGURE 1: Photograph of the dispersions (a), FTIR spectra (b), and UV-vis spectra (c) of CNTs, $\mathrm{Bmim}\left[\mathrm{NiCl}_{3}\right]$, and $\mathrm{CNTs} / \mathrm{Bmim}\left[\mathrm{NiCl} \mathrm{B}_{3}\right]$.

does not exhibit any obvious absorption peak. In contrast, $\mathrm{Bmim}\left[\mathrm{NiCl}_{3}\right]$ only displayed one peak at $508 \mathrm{~nm}$, which resulted from the imidazole ring. The $\mathrm{CNTs} / \mathrm{Bmim}\left[\mathrm{NiCl}_{3}\right]$ curve exhibited the same characteristics as $\mathrm{Bmim}\left[\mathrm{NiCl}_{3}\right]$. However, a slight bathochromic shift of the main absorption peak (from $508 \mathrm{~nm}$ to $513 \mathrm{~nm}$ ) was observed, confirming that a $\pi-\pi$ conjugate force exists between the CNTs and $\operatorname{Bmim}\left[\mathrm{NiCl}_{3}\right]$.

The morphological structure of the $\mathrm{NiO} / \mathrm{CNT}$ composite was investigated using SEM, and $\mathrm{NiO}$ was also investigated for comparison. As shown in Figure 2(a), the NiO/CNTs composite exhibited a uniform morphology, with neither $\mathrm{NiO}$ aggregation nor two-phase ( $\mathrm{NiO}$ and $\mathrm{CNTs}$ ) separation occurring. For comparison, the $\mathrm{NiO}$ synthesized under the same reaction conditions is shown in Figure 2(b). In the absence of CNTs, the $\mathrm{NiO}$ cannot grow on or attach to any substrates; instead, the $\mathrm{NiO}$ adopts an aggregation state. In addition, the more detailed observations presented in Figure 2(a) show that the $\mathrm{NiO}$ nanobeads synthesized in situ nucleated on the one-dimensional (1D) surfaces of the CNTs and eventually grew and fused together to form a thin shell. The uniformly distributed NiO/CNTs composites crosslinked and overlaid each other to form three-dimensional (3D) porous network structures, which indicated that the IL played an important role in the uniform coating of the $\mathrm{NiO}$ nanobeads on the surface of the CNTs. The reactions of ECs are known as an interfacial phenomenon. Thus, the magnitudes of the specific capacitances (SCs) primarily depend on the capacitive properties and microstructures of the electrode materials. According to recent reports, ultrahigh SCs can be obtained when small amounts of metal oxides are uniformly dispersed on carbonaceous substrates with high surface areas and faradaic and carbon electrodes are associated to increase the specific energy $[16,17,21]$. In addition, some researchers believe that the electrochemical performance of ECs with low metal oxide loading is better. However, the SCs of the electroactive material/carbonaceous composites primarily depend on the mass loading and thickness of the electroactive material on the carbonaceous substrate, which allow more electrolyte ions to diffuse into the electroactive material matrix, thereby achieving higher energy storage. Therefore, the deposition of thick electroactive materials on 


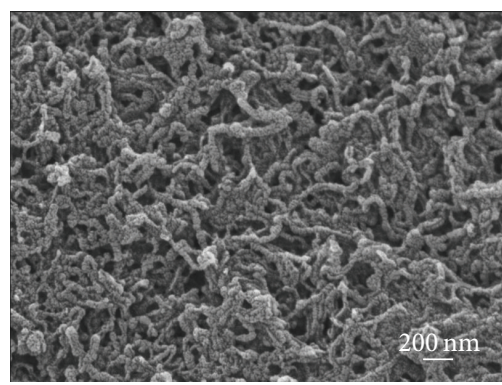

(a)

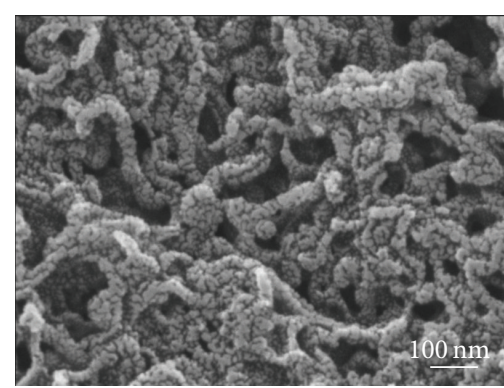

(b)

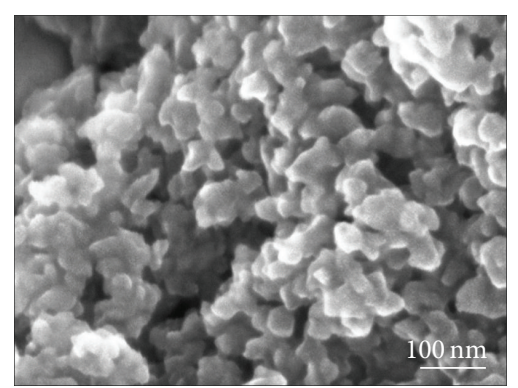

(c)

Figure 2: SEM images of the NiO/CNTs ((a) and (b)) and $\mathrm{NiO}(\mathrm{c})$.

an electronically conductive carbonaceous substrate with a high specific surface area provides a promising approach for improving the SC of metal oxide electrode materials. In addition, according to the SEM observations, this network structure (NiO nanoparticles coated on CNTs to form a 3D morphology) favored the diffusion of electrolyte ions inside the electrode $[9,10,13]$. Furthermore, the electrons and ions must diffuse through the $\mathrm{NiO}$ surfaces or channels rather than through the CNTs. Consequently, the electrochemical performance of the $\mathrm{NiO} / \mathrm{CNTs}$ composite was enhanced. It is reasonable to expect that the $\mathrm{NiO} / \mathrm{CNTs}$ composite synthesized using the noncovalent approach may have good electrochemical properties. Furthermore, the 3D hierarchical architecture of the $\mathrm{NiO} / \mathrm{CNTs}$ composite was observed. These results provide evidence for the successful coating of $\mathrm{NiO}$ on the outer surface of the CNTs and are consistent with the IR and SEM observations.

Figure 3 presents the XRD patterns of the $\mathrm{NiO} / \mathrm{CNTs}$ composite and $\mathrm{NiO}$. The characteristic peak located at $2 \theta=$ $26.4^{\circ}$ corresponds to the hexagonal graphite (002) plane of the CNTs (JCPDS card number 75-1621) [7]. Furthermore, three other well-defined diffraction peaks are observed at $2 \theta$ values of $36.6^{\circ}, 44.6^{\circ}$, and $64.9^{\circ}$, which were successfully identified as the (111), (200), and (220) plane reflections of the $\mathrm{NiO}$ crystalline structure of the $\mathrm{NiO} / \mathrm{CNTs}$ (10 wt.\%). Additionally, these peaks became broader and stronger in the XRD pattern of the $\mathrm{NiO} / \mathrm{CNT}$ composite (as shown in Figure 3). According to the Scherrer formula, the average size of $\mathrm{NiO}$ crystals is about $18 \mathrm{~nm}$. Such a small average size is conducive to the formation of dense packing of $\mathrm{NiO}$ and then fabricates a uniformly heterogeneous oxide layer on the surfaces of CNTs even under high loading.

To evaluate the electrochemical performance, the cyclic voltammograms (CVs) and the galvanostatic charge/ discharge curves of the $\mathrm{NiO} / \mathrm{CNTs}$ electrodes at a current density of $0.5 \mathrm{~A} \mathrm{~g}^{-1}$ are shown in Figure 4 . The $\mathrm{NiO}$ exhibited redox peaks typical of a Faraday capacitor in the voltammogram $[9,10]$. However, the curve of the $\mathrm{NiO} / \mathrm{CNTs}$ electrode was greatly enlarged, indicating that the storage of electrochemical energy mainly resulted from pseudocapacitance by faradaic redox transitions of the $\mathrm{NiO} / \mathrm{CNTs}$ electrodes. With $\mathrm{NiO}$ loading, the $\mathrm{NiO} / \mathrm{CNT}$ s electrode exhibited a better current and a larger SC than that of the $\mathrm{NiO}$ electrode.

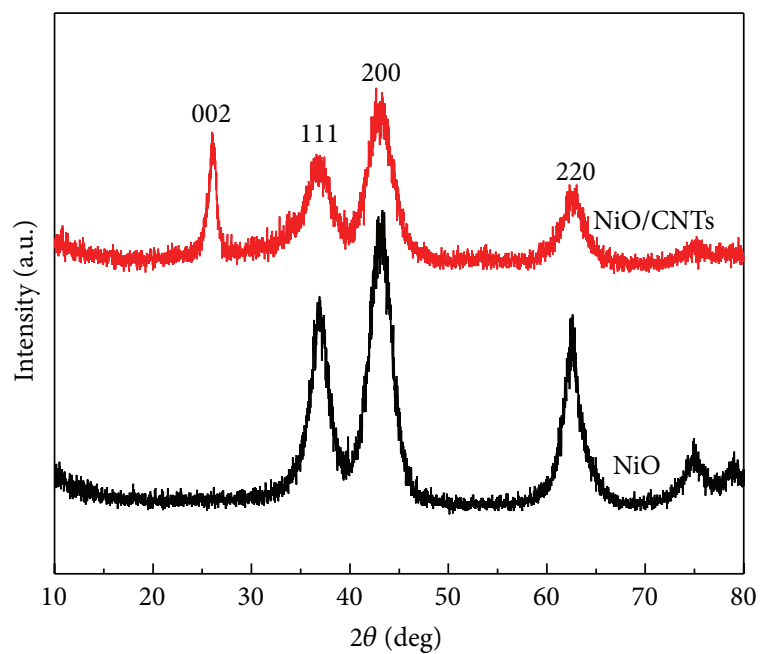

FIGURE 3: XRD patterns of the NiO/CNTs composite and NiO.

In addition, one other reason divides the electrochemical properties of the $\mathrm{NiO} / \mathrm{CNTs}$ electrode. As indicated by the SEM image in Figure 3, NiO loading occurs through $\pi$ $\pi$ interactions between the CNTs and precipitates from $\mathrm{Bmim}[\mathrm{Ni}]^{3+}$ in a noncovalent synthetic process that creates a rough surface area. This $3 \mathrm{D}$ hierarchical architecture of the $\mathrm{NiO} / \mathrm{CNT}$ composite is expected to favor the insertion of electrolytes into (or expulsion out of) the metal oxide matrix, which can increase the contact between the electrolyte and the electrode material and improve the utilization ratio of the $\mathrm{NiO}$. Under heavy mass loading of $\mathrm{NiO}$, it is difficult for the electrolyte ions to diffuse into and encounter the interior of the CNTs because of the concentrated $\mathrm{NiO}$ layer. Hence, $\mathrm{NiO}$ has been reported to generate a high SC due to its rapid and reversible proton intercalation in the lattice $[9,10]$. Figure $4(\mathrm{~b})$ shows the galvanostatic charge/discharge curves of the $\mathrm{NiO}$ electrode. The curve shapes from both electrodes present characteristics that are typical of an ideal pseudocapacitor, which is consistent with the results of the $\mathrm{CV}$ curves. The SC of the composites $\left(C_{m \text {, electrode }}\right)$ and the 


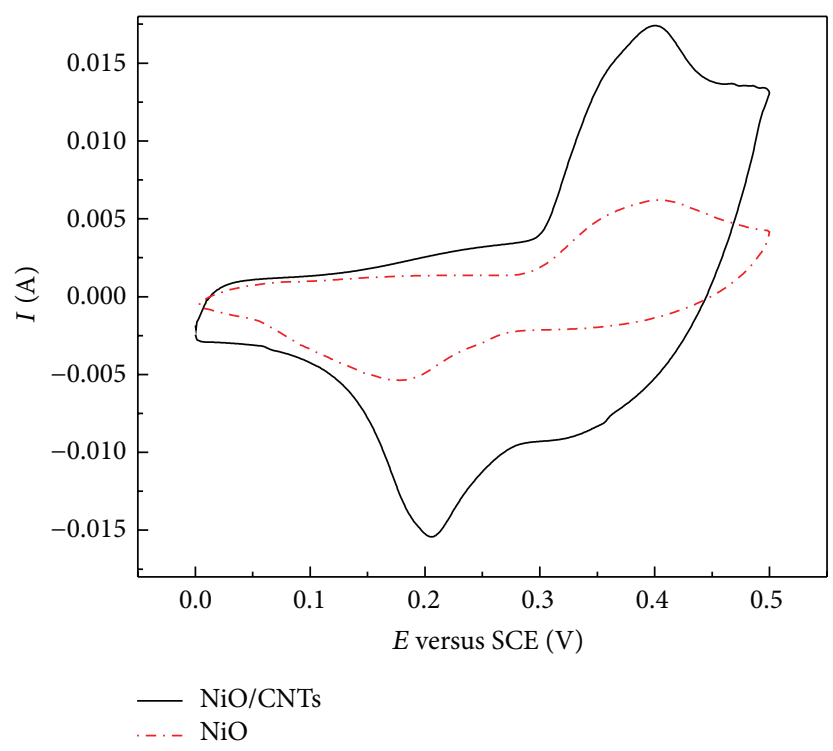

(a)

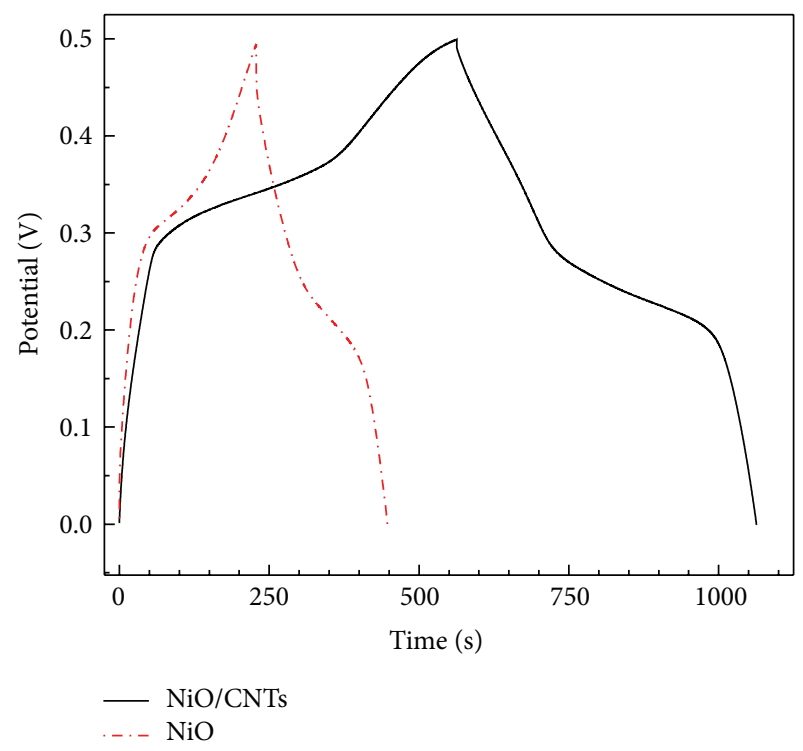

(b)

FIGURE 4: Cyclic voltammograms (a) and charge/discharge curves (b) of the $\mathrm{NiO} / \mathrm{CNTs}$ and $\mathrm{NiO}$ electrodes at potentials of 0 to $0.5 \mathrm{~V}$ with a scan rate of $10 \mathrm{mVs}^{-1}$ and a current density of $0.5 \mathrm{~A} \mathrm{~g}^{-1}$.

use of $\mathrm{NiO}$ in the composites $\left(C_{m}\right)$ were calculated using, respectively,

$$
\begin{gathered}
C_{m}=\frac{C}{m}=\frac{I \times t}{\Delta V \times m} \\
C_{m^{\prime}}=\frac{C}{m^{\prime}}=\frac{I \times \Delta t}{\Delta V \times m \times a},
\end{gathered}
$$

where $I$ is the discharge current, $m$ is the mass of $\mathrm{NiO}$ or $\mathrm{NiO} / \mathrm{CNTs}, \Delta V$ is the potential drop during discharging, $\Delta t$ is the total discharge time, and $C$ is the specific capacitance. In addition, $m^{\prime}$ is the mass of $\mathrm{NiO}$ in the $\mathrm{NiO} / \mathrm{CNTs}$ nanocomposites in one electrode, and a is the wt.\% of $\mathrm{NiO}$ in the $\mathrm{NiO} / \mathrm{CNTs}$. The relationship between the SC and the current density is shown in Figure 5. From these results, we conclude that these types of $\mathrm{NiO} / \mathrm{CNTs}$ composites are characterized by a relatively high SC.

Generally, the SC gradually decreases as the discharging current density increases. At current densities of 0.5 to $4 \mathrm{~A} \mathrm{~g}^{-1}$, the $\mathrm{NiO} / \mathrm{CNTs}$ electrode exhibits higher capacitance retention. Even at a high current density of $4 \mathrm{Ag}^{-1}$, the SCs of the $\mathrm{NiO} / \mathrm{CNT}$ s electrode can remain at $83 \%\left(395 \mathrm{~F} \mathrm{~g}^{-1}\right)$ with a capacity of $0.5 \mathrm{~A} \mathrm{~g}^{-1}$ as a reference. Under identical conditions, the SCs of the $\mathrm{NiO}$ electrode only remain at $103 \mathrm{~F} \mathrm{~g}^{-1}$ and $51 \%$. This good rate capability occurs for various reasons. First, these $\mathrm{NiO} / \mathrm{CNTs}$ composites were constructed with a nanoscale $\mathrm{NiO}$ coating on the CNTs using a noncovalent method, which could not destroy the functional groups on the surfaces of the CNTs. The good electrical conductivity of the $\mathrm{NiO} / \mathrm{CNTs}$ composite provides a "superhighway" for electron hopping in the $3 \mathrm{D}$ conducting matrix. In addition, the unique $3 \mathrm{D}$ morphology of the $\mathrm{NiO} / \mathrm{CNT}$ s composite could reduce the distance for the diffusion of electrons and ions (inner and outer hollow microstructure) and increase

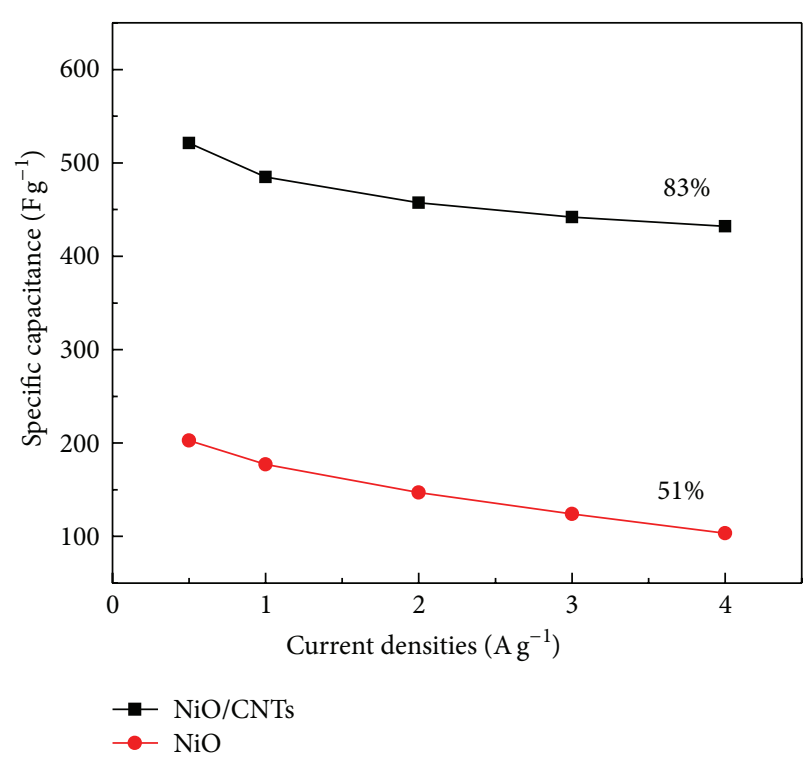

FIGURE 5: The SC values of the $\mathrm{NiO} / \mathrm{CNT}$ and $\mathrm{NiO}$ electrodes at different current densities.

ion transport during rapid charge/discharge reactions $[6,7]$. Furthermore, the three-dimensional (3D) network spatial structures of such electrodes were beneficial for accommodating more volume changes during charging/discharging and could constrain more electrolytes within the electrode. Thus, a higher capacitance retention was achieved. The combination of the above effects improved the rate capability at high current densities $[9,10]$. Hence, even as the current density increases, a large amount of electrolyte ions can access 


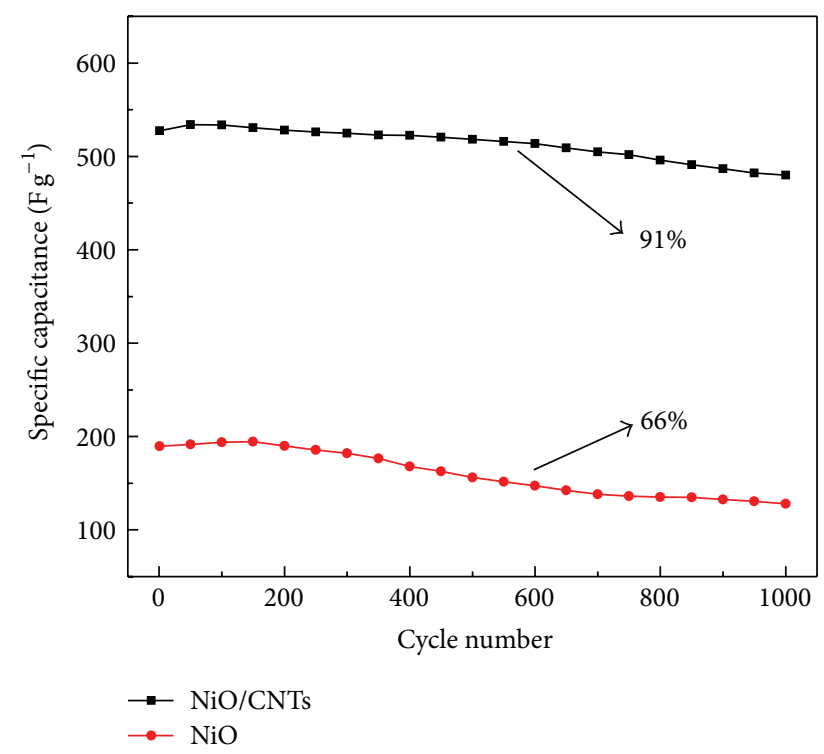

Figure 6: Cycle life data of the $\mathrm{NiO} / \mathrm{CNT}$ and $\mathrm{NiO}$ at a constant current density of $0.5 \mathrm{~A} \mathrm{~g}^{-1}$.

the entrances of the $\mathrm{NiO} / \mathrm{CNT}$ s composite, maintaining a high SC retention ratio.

A long cycle life is another important requirement for practical applications. The $\mathrm{SC}$ of the $\mathrm{NiO} / \mathrm{CNT}$ electrode as a function of the cycle number at a current density of $0.5 \mathrm{Ag}^{-1}$ is presented in Figure 6. As shown, the SC of the $\mathrm{NiO} / \mathrm{CNT}$ electrode only decreased from $527 \mathrm{~F} \mathrm{~g}^{-1}$ to $480 \mathrm{~F} \mathrm{~g}^{-1}$ after 1000 charge/discharge cycles. The SC retention of $91 \%$ indicates that the $\mathrm{NiO} / \mathrm{CNT}$ nanocomposite possesses good electrochemical stability.

\section{Conclusions}

In conclusion, a nanotubular $\mathrm{NiO} / \mathrm{CNT}$ composite was synthesized using an ionic liquid as the reaction medium. The $\mathrm{NiO}$ was loaded on the surface of the CNTs through a $\pi$ $\pi$ conjugate reaction between the imidazole rings of the IL and the benzene rings on the surface of the CNTs $[13,15]$. Because of the noncovalent synthetic process, the nanotubular NiO/CNTs composite exhibits good electronic transmission. Particularly, the unique two-dimensional (2D) hierarchical nanotubular structure and the three-dimensional (3D) porous mesh crossing the structures expose the high specific surface area to the electrolyte, thereby facilitating more electrolyte ions to spread in the electrolyte. The results from the electrochemical tests indicated that the NiO/CNTs composite exhibited a SC of $527 \mathrm{~F} \mathrm{~g}^{-1}$ and a rate capability retention of $91 \%$ in $6 \mathrm{M} \mathrm{KOH}$. Our work not only extends the current solution-based methods to solid-state reactions but also provides the possibility of constructing advanced CNTbased nanotubular electrodes with high SC and excellent rate capability for high-performance electrochemical capacitors. More importantly, the electrode design concept can be easily generalized to other binary or ternary metal oxides with unique nanotubular structures for a broad range of device applications.

\section{Conflict of Interests}

The authors declare that there is no conflict of interests regarding the publication of this paper.

\section{Acknowledgments}

This work was supported by the Xinjiang Key Laboratory of Electronic Information Materials and Devices. In addition, the West Light Foundation of the Chinese Academy of Sciences (no. XBBS201111) and the National Natural Science Foundation of China (no. 21103225) are acknowledged.

\section{References}

[1] R. R. Salunkhe, S.-H. Hsu, K. C. W. Wu, and Y. Yamauchi, "Large-scale synthesis of reduced graphene oxides with uniformly coated polyaniline for supercapacitor applications," ChemSusChem, vol. 7, no. 6, pp. 1551-1556, 2014.

[2] N. L. Torad, R. R. Salunkhe, Y. Li et al., "Electric double-layer capacitors based on highly graphitized nanoporous carbons derived from ZIF-67," Chemistry, vol. 20, no. 26, pp. 7895-7900, 2014.

[3] R. R. Salunkhe, B. P. Bastakoti, C.-T. Hsu et al., "Direct growth of cobalt hydroxide rods on nickel foam and its application for energy storage," Chemistry, vol. 20, no. 11, pp. 3084-3088, 2014.

[4] C. Shang, S. Dong, S. Wang et al., "Coaxial $\mathrm{NixCo}_{2 x}(\mathrm{OH})_{6 x} / \mathrm{TiN}$ nanotube arrays as supercapacitor electrodes," ACS Nano, vol. 7, no. 6 , pp. 5430-5436, 2013.

[5] B. Cui, H. Lin, J.-B. Li, X. Li, J. Yang, and J. Tao, "Core-ring structured $\mathrm{NiCo}_{2} \mathrm{O}_{4}$ nanoplatelets: synthesis, characterization, and electrocatalytic applications," Advanced Functional Materials, vol. 18, no. 9, pp. 1440-1447, 2008.

[6] M. Alvaro, P. Atienzar, P. De La Cruz, J. L. Delgado, H. Garcia, and F. Langa, "Sidewall functionalization of singlewalled carbon nanotubes with nitrile imines. Electron transfer from the substituent to the carbon nanotube," The Journal of Physical Chemistry B, vol. 108, no. 34, pp. 12691-12697, 2004.

[7] J. Y. Lee, K. Liang, K. H. An, and Y. H. Lee, "Nickel oxide/carbon nanotubes nanocomposite for electrochemical capacitance," Synthetic Metals, vol. 150, no. 2, pp. 153-157, 2005.

[8] C. Yuan, J. Li, L. Hou, X. Zhang, L. Shen, and X. W. Lou, "Ultrathin mesoporous $\mathrm{NiCO}_{2} \mathrm{O}_{4}$ nanosheets supported on $\mathrm{Ni}$ foam as advanced electrodes for supercapacitors," Advanced Functional Materials, vol. 22, no. 21, pp. 4592-4597, 2012.

[9] C. Yuan, S. Xiong, X. Zhang et al., “Template-free synthesis of ordered mesoporous $\mathrm{NiO} /$ poly(sodium-4-styrene sulfonate) functionalized carbon nanotubes composite for electrochemical capacitors," Nano Research, vol. 2, no. 9, pp. 722-732, 2009.

[10] B. Gao, C. Yuan, L. Su, S. Chen, and X. Zhang, "High dispersion and electrochemical capacitive performance of $\mathrm{NiO}$ on benzenesulfonic functionalized carbon nanotubes," Electrochimica Acta, vol. 54, no. 13, pp. 3561-3567, 2009.

[11] C.-T. Hsieh, H. Teng, W.-Y. Chen, and Y.-S. Cheng, "Synthesis, characterization, and electrochemical capacitance of aminofunctionalized carbon nanotube/carbon paper electrodes," Carbon, vol. 48, no. 15, pp. 4219-4229, 2010. 
[12] B. A. Kakade and V. K. Pillai, "An efficient route towards the covalent functionalization of single walled carbon nanotubes," Applied Surface Science, vol. 254, no. 16, pp. 4936-4943, 2008.

[13] B. Wu, D. Hu, Y. Kuang, B. Liu, X. Zhang, and J. Chen, "Functionalization of carbon nanotubes by an ionic-liquid polymer: dispersion of $\mathrm{Pt}$ and $\mathrm{PtRu}$ nanoparticles on carbon nanotubes and their electrocatalytic oxidation of methanol," Angewandte Chemie International Edition, vol. 48, no. 26, pp. 4751-4754, 2009.

[14] J. Zou, J. Liu, A. S. Karakoti et al., "Ultralight multiwalled carbon nanotube aerogel," ACS Nano, vol. 4, no. 12, pp. 7293-7302, 2010.

[15] C. Zhong, T. Sasaki, A. Jimbo-Kobayeshi et al., "Syntheses, structures, and properties of a series of metal ion-containing dialkylimidazolium ionic liquids," Bulletin of the Chemical Society of Japan, vol. 80, no. 12, pp. 2365-2374, 2007.

[16] T.-Y. Wei, C.-H. Chen, H.-C. Chien, S.-Y. Lu, and C.-C. Hu, "A cost-effective supercapacitor material of ultrahigh specific capacitances: spinel nickel cobaltite aerogels from an epoxidedriven sol-gel process," Advanced Materials, vol. 22, no. 3, pp. 347-351, 2010.

[17] H. Li, J. K. Jo, L. Zhang, C.-S. Ha, H. Suh, and I. Kim, "A general and efficient route to fabricate carbon nanotube-metal nanoparticles and carbon nanotube-inorganic oxides hybrids," Advanced Functional Materials, vol. 20, no. 22, pp. 3864-3873, 2010.

[18] R. H. Baughman, A. A. Zakhidov, and W. A. de Heer, "Carbon nanotubes - the route toward applications," Science, vol. 300, pp. 2072-2074, 2003.

[19] X. Liu, R. Ma, Y. Bando, and T. Sasaki, "A general strategy to layered transition-metal hydroxide nanocones: tuning the composition for high electrochemical performance," Advanced Materials, vol. 24, no. 16, pp. 2148-2153, 2012.

[20] T. Fukushima and T. Aida, "Ionic liquids for soft functional materials with carbon nanotubes," Chemistry-A European Journal, vol. 13, no. 18, pp. 5048-5058, 2007.

[21] S. Dai, Y. H. Ju, H. J. Gao, J. S. Lin, S. J. Pennycook, and C. E. Barnes, "Preparation of silica aerogel using ionic liquids as solvents," Chemical Communications, no. 3, pp. 243-244, 2000. 

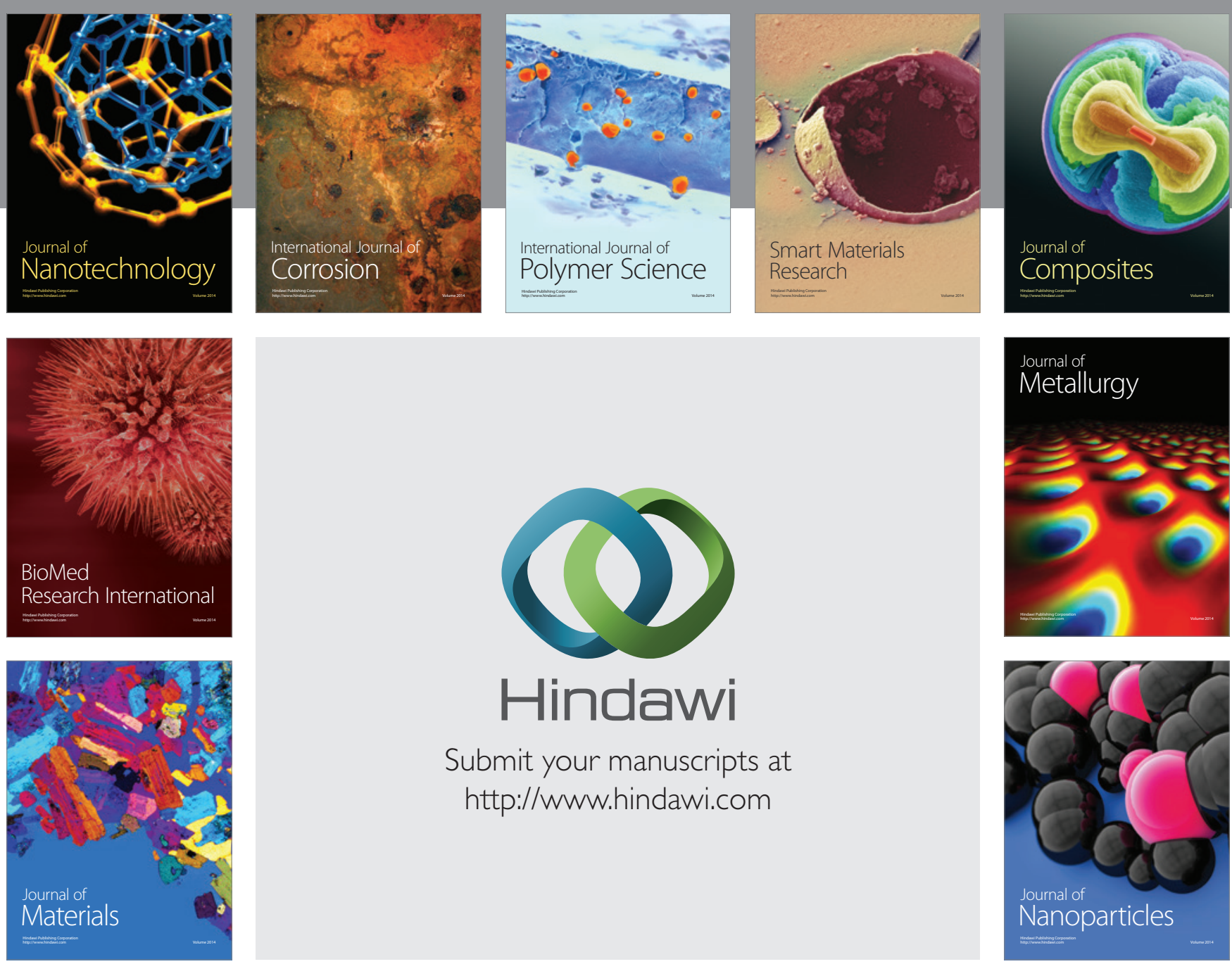

Submit your manuscripts at http://www.hindawi.com
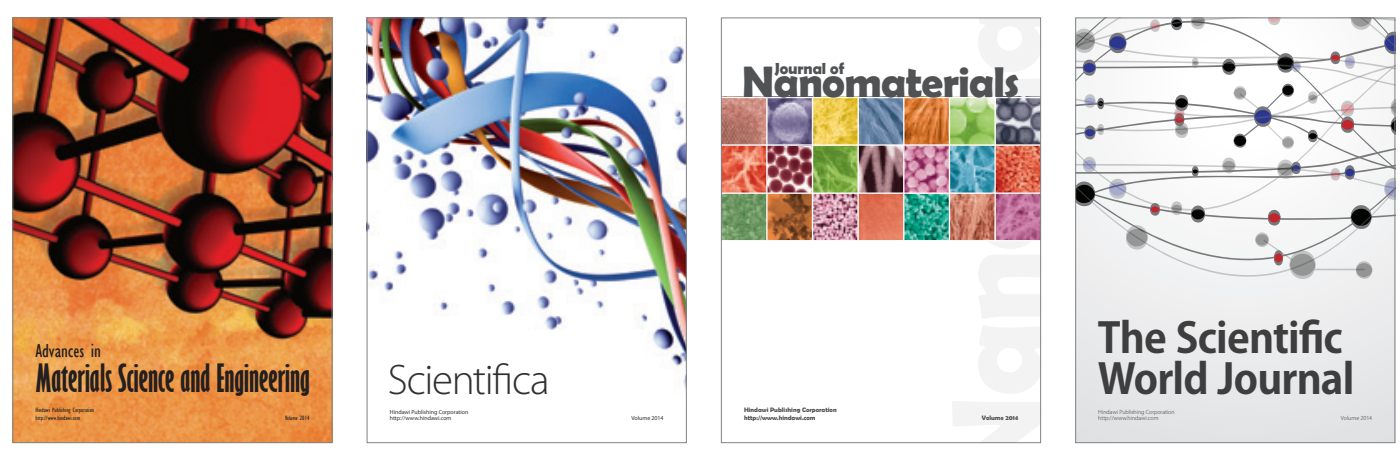

\section{The Scientific World Journal}
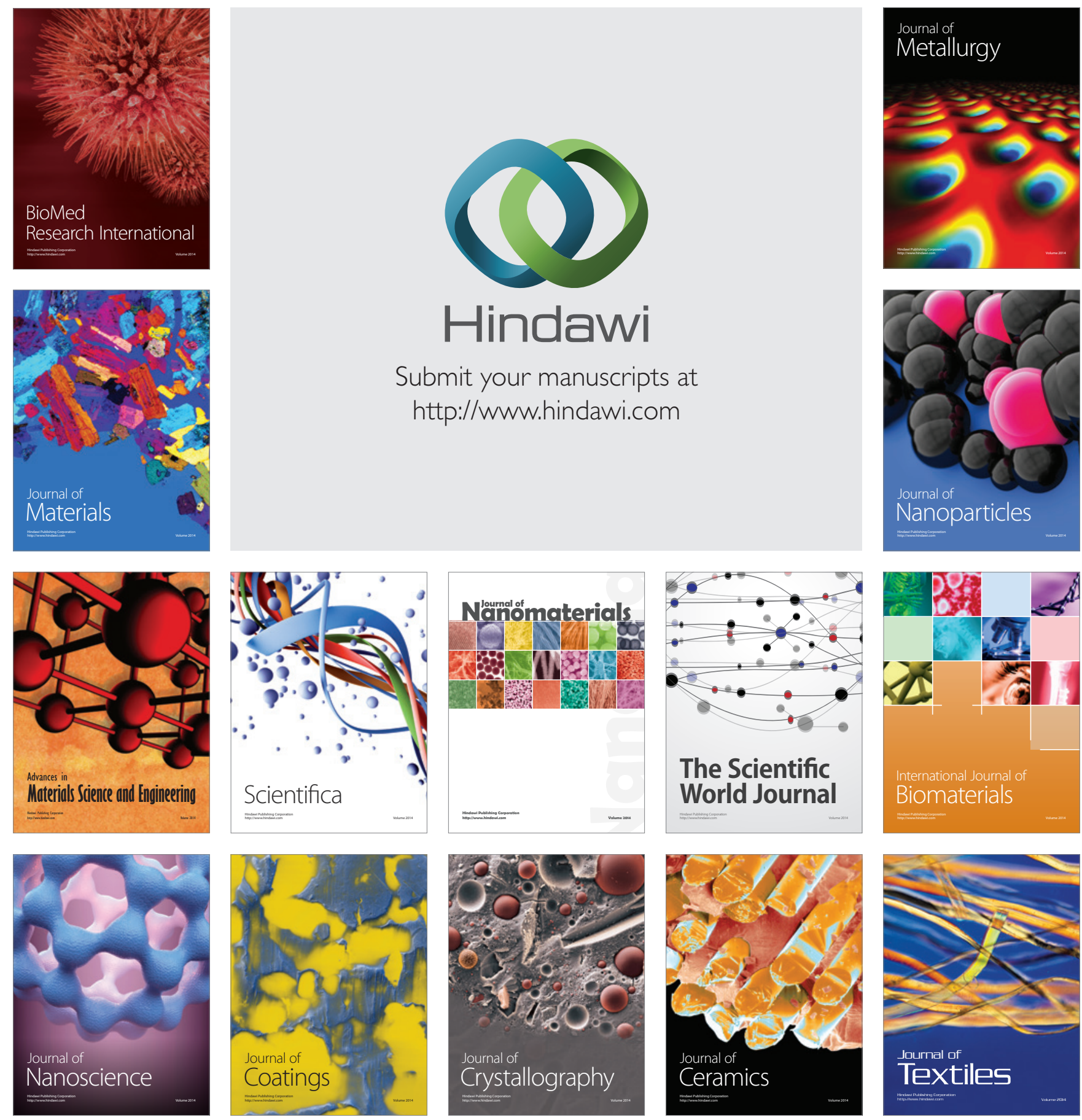